\title{
Motor activity of nursing students in the conditions of the COVID-19 pandemic
}

\author{
Nikoleta Popova ${ }^{1}$, Anna Georgieva ${ }^{1, *}$, and Vasilena Digalovska ${ }^{1}$ \\ ${ }^{1}$ Medical University "Prof. Dr.Paraskev Stoyanov", Department of Nursing care, Varna, Bulgaria
}

\begin{abstract}
The COVID-19 pandemic has changed all areas of human life and activity. A number of lifestyle changes associated with health risk factors have occurred, one of which is inactivity. The purpose of this study is to investigate the opinion of nursing students about their motor activity in the conditions of the COVID-19 pandemic. Material and methods: In our report, we have applied analysis of literary sources and a survey (on-line anonymous group survey). The opinion of 60 nursing students of second, third and fourth year at the Medical University - Varna was studied. The nurses received explanation for the purpose and methodology of the study, their anonymity was guaranteed, and their informed consent for the study was obtained. The study was conducted in August 2020-January 2021. Graphical analysis was used to visualize the observed processes and phenomena. To create the graphs, we used Microsoft Office Excel 2013. The data were processed using the IBM SPSS Statistics 19 statistical package. Results: The majority of the respondents shared that they had been less active compared to the time before the pandemic began. The search for alternatives for exercising and sports among students is hampered by a number of factors, which relate to the changes in the organization of life and educational activities associated with measures to stop the pandemic.

Conclusion: The results of this study prove that the COVID-19 pandemic has had an impact on the lifestyle of the nursing students - most of them have reduced their motor activity. The data highlights the need to find alternatives and strategies to reduce the inactivity.
\end{abstract}

Key words - motor activity, nurses, students, pandemic.

\section{Introduction}

The COVID-19 pandemic, also known as the coronavirus pandemic, was first identified in December 2019 in Wuhan, China. The World Health Organization declared the outbreak a Public Health Emergency of International Concern in January 2020 and a pandemic in March 2020. As of 13 March 2021, more than 119 million cases have been confirmed, with more than 2.64 million deaths attributed to COVID-19, making it one of the deadliest pandemics in history [1].

The COVID-19 pandemic has changed all areas of human life and activity. Along with the threat to human life and health caused by the COVID-19 infection itself, there is a number of lifestyle changes associated with health risk factors, one of which is inactivity.

\footnotetext{
*Corresponding author: anngebg@gmail.com
} 
The COVID-19 pandemic means that many of people are staying at home and sitting down more than we usually do. It is hard for many people to do the sort of exercise they would normally do. It is even harder for people who do not usually do a lot of physical exercise. But at a time like this, it is very important for people of all ages and abilities to be as active as possible. WHO's Be Active campaign aims to help people do just that - and to have some fun at the same time [1].

WHO defines physical activity as any bodily movement produced by skeletal muscles that requires energy expenditure. Physical activity refers to all movement including during leisure time, for transport to get to and from places, or as part of a person's work. Both moderate and vigorous physical activities improve health [2].

Regular physical activity is proven to help prevent and manage non-communicable diseases such as heart disease, stroke, diabetes and several cancers. It also helps prevent hypertension, maintain healthy body weight, and can improve mental health, quality of life and wellbeing [1-3].

In connection with maintaining optimal physical activity and protecting health at home, the WHO makes the following recommendations:

- Taking regular breaks between work involving prolonged sitting. During these breaks, physical activity of varying intensity is recommended.

- Maintaining proper posture.

- Performing stretching and endurance exercises, climbing stairs, dancing, playing with children, gardening, using Internet resources with exercises at home, etc.

- for healthy adults, at least 30 minutes of physical activity per day is recommended, and for children, at least one hour per day [1].

In addition, the WHO provides a number of examples of physical activity, such as online exercise classes, dancing, playing active video games, jumping rope, and practising muscle strength and balance training [1]. The need to recognize and maintain physical activity as a model of health behaviour for people of different ages underlies in the WHO Global Plan of Action for Physical Activity 2018-2030: More Active People for a Healthier World and has been proven by a number of studies [3-10]. In response to stimulating support for motor activity, during the pandemic, the European Commission has launched challenge "Be active at home". The initiators are inviting social media users to share their "recipes" for physical activity under quarantine conditions introduced in many countries around the world due to the COVID-19 coronavirus epidemic [11].

\section{Objectives}

Our objective is to study the opinion of nursing students about their motor activity in the conditions of the COVID-19 pandemic.

To achieve this goal, the following tasks are set:

- to investigate changes in the motor activity of student nurses during the Covid 19 pandemic; - to study the socio-demographic characteristics of student nurses, such as age, gender, Body Mass Index (BMI);

- to study the relationship and influence of the socio-demographic characteristics of student nurses on their motor activity in the context of the Covid 19 pandemic;

- to study the approaches of the students in maintaining their physical activity in the conditions of the Covid pandemic;

- to study the difficulties of the students in maintaining their physical activity and the factors determining them. 


\section{Material and methods}

In our report, we have applied analysis of literature sources and a survey (on-line anonymous group survey). The opinion of 60 nursing students of second, third and fourth year at the Medical University - Varna was studied. The nurses received explanation about the purpose and methodology of the study. Their anonymity was guaranteed, and their informed consent for the study was obtained. The study was conducted in August 2020-January 2021. Graphical analysis was used to visualize the observed processes and phenomena. To create the graphs, we used Microsoft Office Excel 2013. The data was processed using the IBM SPSS Statistics 19 statistical package.

\section{Results}

Most of the participants in our study were in the age group of 19-24 years (83.5\%), followed by $25-34$ years $(3.3 \%)$ and $35-44$ years $(13.2 \%)$ (Fig. 1).

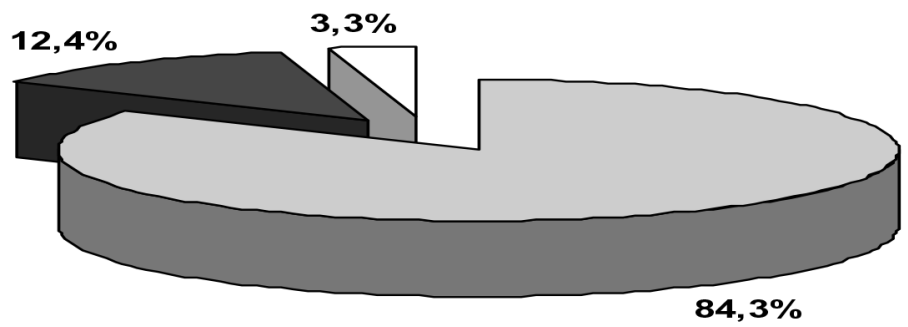

$\square$ 19-24 $\square$ 25-34 $\square$ 35-44

Figure 1. Age (years) of participant.

As can be seen from the data in Fig. 2, almost all participants in the study were women $(97.6 \%)$.

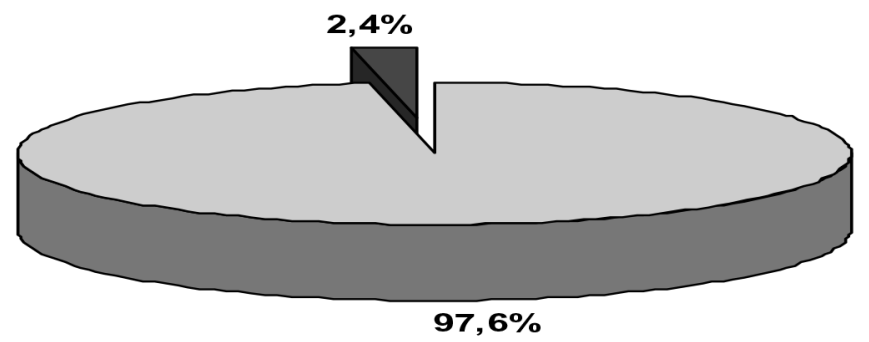

$\square$ women $\square$ men

Figure 2. Gender of participants.

The majority of student nurses are of normal weight (83.3\%), more than one tenth of them are with pre-obesity (11.7\%). Only one student is underweight and two are with obesity. 
The COVID-19 pandemic had an impact on the physical activity of the majority of students (73.3\%). Only $26.7 \%$ were able to keep it at the same level (Fig. 3).

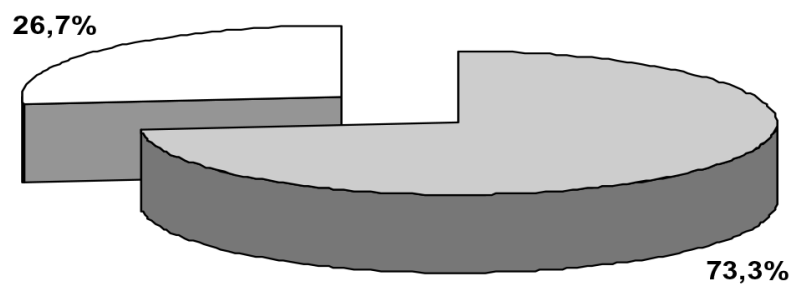

$\square$ yes $\square$ no

Figure 3. The opinion of the respondents for the question: Does the COVID-19 pandemic affect your physical activity?

No statistically significant relationship was found between the age, sex, and BMI of the students and the change in their motor activity in the conditions of the Covid-19 pandemic $(p>0.05)$.

Most respondents spend less than 30 minutes a day on physical activity (48.3\%). The remaining $28.3 \%$ of students exercise 60 minutes a day and approximately a quarter of the respondents 30 minutes a day $(28.3 \%)$.

The BMI of the respondents is significantly inversely related to the time they spend during the day to moves the time for physical activity decreased, the BMI of the study participants increased (Gamma $\square=-0.623 ; p<0.005$ ).

Most of the respondents, who managed to maintain their physical activity in the conditions of the COVID-19 pandemic, have not changed their habits. They continue to walk outside as before (38.5\%), approximately a quarter continue to play sports at home, as before (23.1\%), and the rest have replaced sports in a gym with long walks outside (15.4\%), with sports outside $(7.7 \%)$ and with training at home (7.7\%). For a small number of students, the lack of change is due to the fact that even before the pandemic they have had a low physical activity (7.7\%), (Fig. 4).

There was no statistically significant correlation between participants' age, sex, and BMI and the ways, in which they were able to maintain their motor activity in the conditions of the Covid-19 pandemic $(p>0.05)$.

In a significant part of the students the physical activity decreased (87.0\%) and in an insignificant part of them it increased (13.0\%), (Fig. 5).

Based on the statistical analysis, a complete relationship between the age of the students and the change in their motor activity due to the Covid-19 pandemic was proved (Gamma $\square$ $=1.00, p<0.001$ ). All students who indicated no change in physical activity are in the age group 19-24 years, while all representatives of the age groups 25-34 years and 35-44 years confirm the presence of a change in their motor activity. The influence of the students' gender and BMI on changes in their motor activity in the Covid-19 pandemic was not demonstrated $(p>0.05)$. 


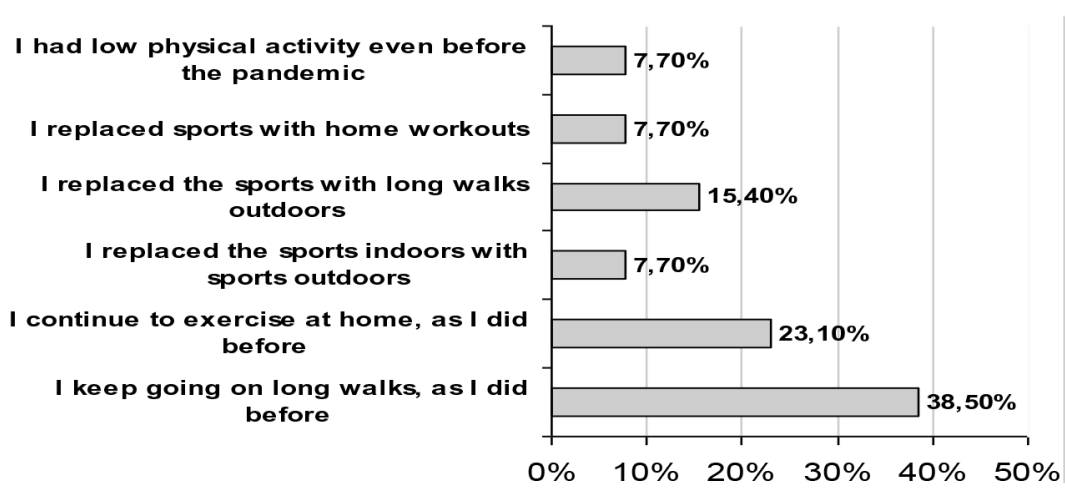

Figure 4. The opinion of the respondents for the question: How did you manage to keep your physical activity the same without being affected by the COVID-19 pandemic?

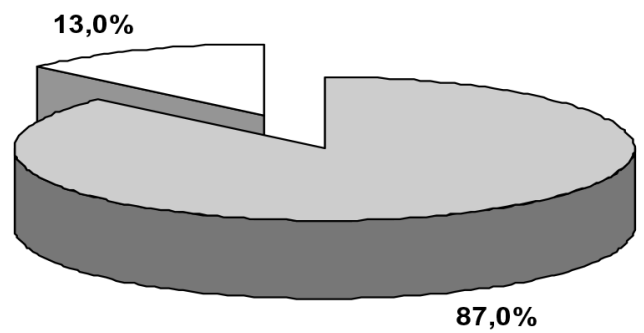

$\square$ my physical activity decreased $\square$ my physical activity increased

Figure 5. The opinion of the respondents for the question: How did COVID-19 affect your physical activity?

A significant proportion of study participants whose physical activity decreased because of the pandemic tried to look for alternatives to its previous size (79.2\%). Only one fifth of the respondents say that they do not make such attempts (20.8\%), (Fig. 6).

The respondents' opinion on attempts to find alternatives to restore their physical activity did not statistically depend on their age, gender, and BMI $(p>0.05)$.

Those looking for alternatives to increase their physical activity have opted for replacing sports in a gym with long walks outside $(60.0 \%)$, with training at home $(55.0 \%)$ and with sports outside $10.0 \%$, (Fig. 7).

The results exceed $100 \%$, as the respondents had the opportunity to indicate more than one answer.

Difficulties in finding alternatives for maintaining the previous physical activity in the conditions of COVID-19 pandemic are experienced by $40 \%$ of the respondents, while for the rest this is not a significant problem (60.0\%) (Fig. 8.).

The influence of the students' age, gender, and BMI on the presence of difficulties in maintaining their motor activity in the conditions of the Covid-19 pandemic was not found $(p>0.05)$. At the same time, a strong proportional correlation was demonstrated between 


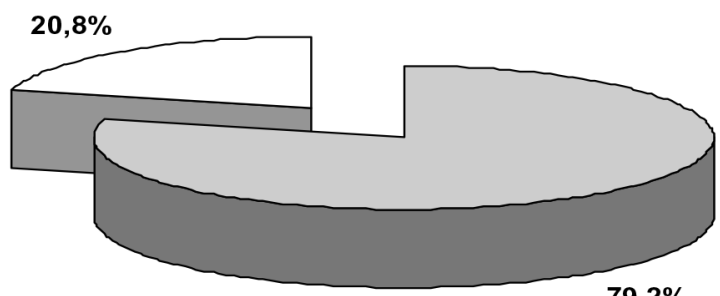

$\square$ yes $\square$ no

Figure 6. The opinion of the respondents for the question: Are you trying to find alternatives to restore your physical activity?

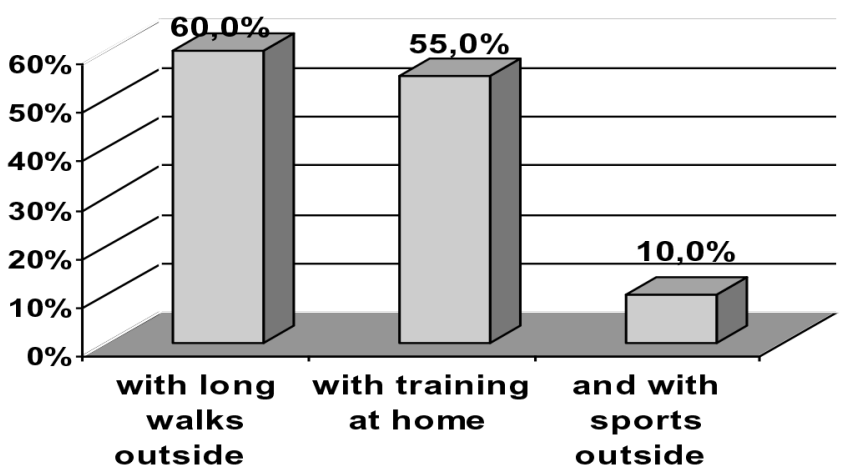

Figure 7. Alternatives to increase physical activity of nursing students.

the difficulties associated with the Covid-19 pandemic, negatively affecting the motor activity of the respondents and the alternatives sought by students for maintaining physical activity (Phi $=0.927 ; p<0.001)$. Those of them who have managed to find alternatives have no difficulty in maintaining their motor activity at the previous level.

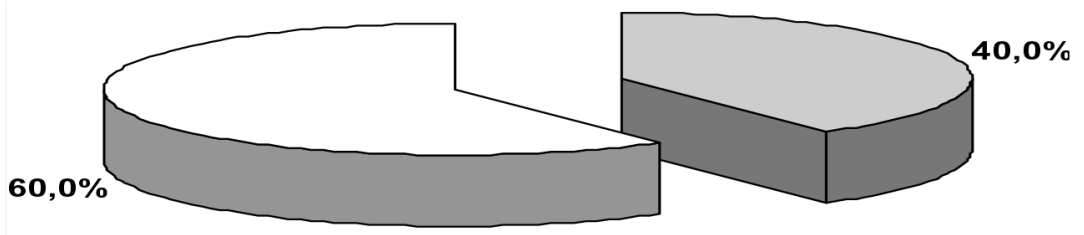

\section{$\square$ yes $\square$ no}

Figure 8. The opinion of the respondents for the question: Do you have difficulty finding alternatives to restore your physical activity to its previous form before the pandemic?

The main factors due to the difficulties in maintaining physical activity during the pandemic, according to more than half of the students, are the measures related to the change 
in the organization of education in electronic form (57.1\%) and the measures related to the closure of sports facilities (35.7\%) (Fig. 9.).

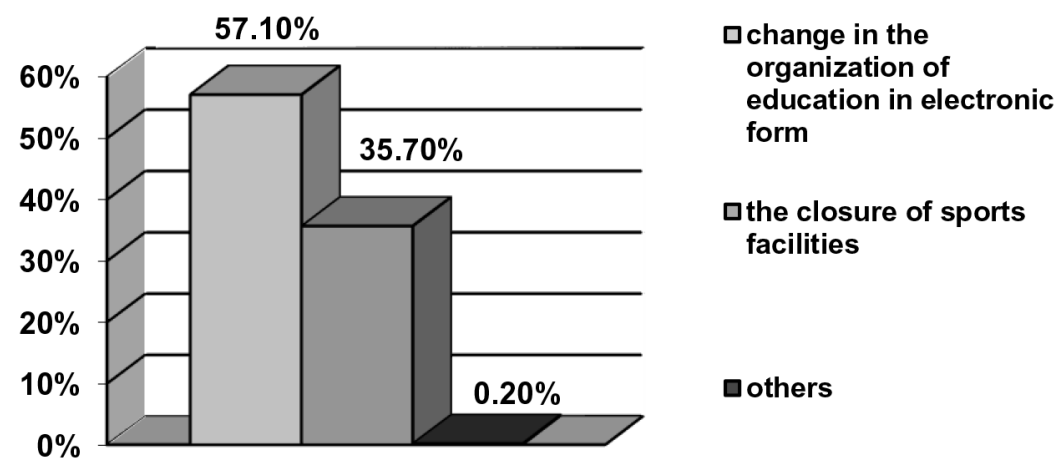

Figure 9. Factors due to the difficulties in maintaining physical activity.

The students' perceptions of the main factors associated with the Covid-19 pandemic, which negatively affected their motor activity, correlated significantly in direct proportion to their BMI (Gamma $\square=0.781 ; p<0.041$ ). All students who indicated that nothing hindered their physical activity had a normal BMI, while those who indicated difficulties arising from "change in the organization of education in electronic form" and "the measures related to the closure of sports facilities" are dominated by those who are overweight and obese. These results are the basis for future in-depth studies aimed at the interdependence between the BMI of nursing students, their motor activity, and the factors that negatively affect it.

\section{Discussion}

Similar to our study, the studies by other researchers were conducted during the same period. For example, an Italian study involving 398 students used the International Physical Activity Questionnaire to assess physical activity and sedentary behaviour during COVID-19 blockade in the spring of 2020. Similar to the results of our study, the data from the Italian study prove that the sedentary behaviour of the respondents during blocking is greater than before the blocking ( $p=0.003$ ). On the other hand, the data of the Italian study, in contrast to the results of our study show an increase in physical activity of some groups, as well as differences in gender, year of study, BMI, based on group analysis [12]. Based on data from its study, Barkley J. et al. (2020) conclude that, while the closure of the university increased sedentary behaviour in the sample, it only reduced the physical activity of the participants who were most active before the blockade [13]. The proportion of inactive or under-active individuals in our study is comparable to data from studies by Sallis J.F. et al. (2020) and Taeymans J. et al. (2021) [14, 15]. According to the results of the study by Sallis J.F. et al. (2020), the observed difference between these two study groups is comparable to a short walking tour of 15 minutes for 5 days a week. According to the authors, when summarized over time, even small differences can become clinically significant. The importance of physical activity is known [14]. In this regard, Sallis J.F. et al. (2020) emphasize that active muscles produce chemicals that improve the functioning of the immune system, which in turn reduces the rate of infections and reduces inflammation, and these are the main causes of lung damage from SARS-CoV-2 infection [14]. 
In another study, $4.6 \%$ of respondents were classified as inactive, $32.5 \%$ as minimally active and $62.9 \%$ as health improving physically active. Individuals classified as belonging to the latter group have participated in various manifestations of physical activity with evidence of health-enhancing effects [16]. These results are lower than those from the Swiss Health survey in 2017 are where the share of trained and sufficiently active people who meet the recommendations for physical activity is $76 \%$ [17].

Another study aimed to assess changes in obesity and patterns of activity among young people during the blockade of the pandemic in China. The results of this study show that significant changes have occurred, including decreased physical activity and increased sedentary, sleep and screen time [18]. Similar to our results are data from a study conducted in Saudi Arabia, according to which most students report poor physical activity during the COVID-19 pandemic and most of them lead a sedentary lifestyle [19].

\section{Conclusions}

A significant proportion of nursing students confirm that the COVID-19 pandemic has affected their motor activity. More than half of the study participants told that they had been less active compared to the time before the pandemic began. The search for alternatives for exercising and sports among students is hampered by a number of factors, which relate to the changes in the organization of life and educational activities associated with measures to stop the pandemic. The results of this study prove that the COVID-19 pandemic has had an impact on the lifestyle of the nursing students - most of them have reduced their motor activity. The data highlights the need to find alternatives and strategies to reduce the inactivity. A complete relationship between the age of the students and the change in their motor activity due to the Covid-19 pandemic was proved. All students who indicated no change in physical activity are in the age group 19-24 years, while all representatives of the age groups 25-34 years and 35-44 years confirm the presence of a change in their motor activity. The influence of students' gender and BMI on changes in their motor activity in the Covid-19 pandemic was not demonstrated.

The BMI of the respondents is significantly inversely related to the time they spend during the day to moving; when the time for physical activity decreased, the BMI of the study participants increased.

The results of this study allow recommendations to be made for the development of programs and action plans for better preparation of campaigns to promote the health of students during the pandemic period, as well as if a lockdown period is imposed in the future.

\section{References}

[1] WHO. Healthy at Home - Physical activity. Available: https://www.who.int/ news-room/campaigns/connecting-the-world-to-combatcoronavirus/healthyathome/ healthyathome---physical-activity

[2] WHO. Physical activity. Available: https://www.who.int/news-room/factsheets/detail/physical-activity

[3] WHO. Global action plan on physical activity 2018-2030: more active people for a healthier world.Available: https://www.who.int/publications/i/item/9789241514187

[4] S. Filkova, Tsv. Tarpomanova, Raising the parents' awareness of the importance of the physical activity for the children health, Activities in Physical Education and Sport 6(1), 25-28 (2016) 
[5] S. Filkova, V. Todorova, V. Kovacheva, Need for awareness of physical activity as a model of health behaviour, Varna Medical Forum 2(2), 37-40 (2013)

[6] S. Filkova, S. Bogomilova, Tsv. Tarpomanova, Study of the motor activity of obese children, Varna Medical Forum 3(4), 49-52 (2015)

[7] Y. Markova, J. Pavlovska, Analysis of the needs for care of the elderly in Bulgaria, Proceedings of the XII National Forum of Health Care Specialists, 11 - 12. 11. 2016, Shumen, Ed. S. Toncheva 1, 101-105 (2016)

[8] Y. Markova, S. Borisova, J. Pavlovska, Study of the need for medical and social care in the home for the elderly and old people, Varna Medical Forum 7(4), 186-190 (2018)

[9] Kr. Stamova, St. Staykova, R. Dimitrova, Study of the need to provide help and support to patients on hemodialysis treatment from the dialysis clinic at the Multi-profile Hospital for Active Treatment "St. Marina" - Varna,Varna Medical Forum 3(2), 308-311 (2014)

[10] Z. Zdravkova-Chaush, N. Mikhailova, K. Petrova, Kr. Stamova, The need to obtain additional health information of patients with cardiovascular diseases and their families, Varna Medical Forum 6(2), 157-160 (2017)

[11] Euro pulse. The European Commission has launched a challenge to keep fit at home during quarantine. April 8 (2020)

[12] L. Di Renzo, P. Gualtieri, F. Pivari, et al., Eating habits and lifestyle changes during COVID-19 lockdown: an Italian survey. J Transl Med. 18(1), 229, DOI:10.1186/s12967-020-02399-5, (2020)

[13] J. Barkley, A. Lepp, E. Glickman, G. Farnell, J. Beiting, Wiet R, et al, The acute effects of the COVID-19 pandemic on physical activity and sedentary behavior in university students and employees, Int J Exerc Sci 13(5), 1326-1339 (2020)

[14] J.F. Sallis, D. Adlakha, A. Oyeyemi, D. Salvo. An international physical activity and public health research agenda to inform coronavirus disease-2019 policies and practices. J Sport Health Sci Jul 9(4), 328-334 (2020)

[15] J. Taeymans, E. Luijckx, S. Rogan, K. Haas, H. Baur, Physical Activity, Nutritional Habits, and Sleeping Behavior in Students and Employees of a Swiss University During the COVID-19 Lockdown Period: Questionnaire Survey Study, JMIR Public Health Surveill 7(4), e26330. Available: https://publichealth.jmir.org/2021/4/e26330, DOI: $10.2196 / 26330(2021)$

[16] IPAQ Group. Guidelines for Data Processing and Analysis of the International Physical Activity Questionnaire (IPAQ). 2005 Nov. Available: $\quad$ https://docs.google.com/viewer? $\mathrm{a}=\mathrm{v} \backslash \&$ pid=sites $\backslash \&$ srcid= ZGVmYXVsdGRvbWFpbnx0aGVpcGFxfGd4OjE0NDgxMDk3NDU1YWRIZTM [accessed 2021-03-11]

[17] Schweizerische Gesundheitsbefragung 2017. Neuchâtel, Switzerland: Bundesamt für Statistik, Available: https://www.bfs.admin.ch/bfs/de/home/statistiken/gesundheit/ erhebungen/sgb.html [accessed 2021-03-11] (2017)

[18] S. Yang, B. Guo, L. Ao, et al. Obesity and activity patterns before and during COVID-19 lockdown among youths in China. Clin Obes 10(6), e12416, DOI:10.1111/cob.12416. (2020)

[19] S.M. Jalal, M.R.M. Beth, H.J.M. Al-Hassan, N.M.J. Alshealah, Body Mass Index, Practice of Physical Activity and Lifestyle of Students During COVID-19 Lockdown, J Multidiscip Healthc 14, 1901-1910. Available: https://doi.org/10.2147/JMDH.S325269 (2021) 